\title{
Managing the Complications of Cirrhosis during COVID-19 Pandemic: Review of the Literature and a Case Study
}

H.Elabbassi ${ }^{1 *}$, K. Krati ${ }^{1}$, H. Aouroud ${ }^{1}$, Oubaha $S^{2}$, Samlani $Z^{1}$

${ }^{1}$ Department of hépato-gastro-entérology, Arrazi Hospital, Marrakech VI Hospital Center, Marrakech, Morocco

${ }^{2}$ Department of Physiology, Faculty of Medicine and Pharmacy Cadi ayyad, Marrakech, Morocco

DOI: $10.36347 /$ sjmcr.2021.v09i03.011

| Received: 03.02.2021 | Accepted: 17.02.2021 | Published: 13.03.2021

*Corresponding author: Hanane El Abbassi

\section{Abstract}

The COVID-19 pandemic has spread rapidly through the world and the care system should adapt rapidly to this context. In our case, managing cirrhosis and its complications during this pandemic situation is one of the main challenges we are facing actually. For this reason, we report in this article the case of a patient admitted in compensation for his cirrhosis and diagnosed positive COVID -19. First, we explained the adopted protocol. Then, we reviewed the main protocols proposed for similar cases in the literature.

Keywords: Complications, Cirrhosis, COVID-19 Pandemic.

Copyright $\odot 2021$ The Author(s): This is an open-access article distributed under the terms of the Creative Commons Attribution 4.0 International License (CC BY-NC 4.0) which permits unrestricted use, distribution, and reproduction in any medium for non-commercial use provided the original author and source are credited.

\section{INTRODUCTION}

In December 2019, a novel coronavirus responsible for respiratory tract infections (COVID-19, known also as SARS-CoV-2) emerged in in Wuhan, China. This virus has changed the world and created several challenges to healthcare services; it's consequences on social life, economics, and an infrastructure has made it a serious health problem. The major risk came from the high transmission efficiency from human-to-human of the coronavirus. Many scientists [1-4] had explained this by the capacity of the pathogens from the virus to survive in the air for up to three (3) hours, for twenty-four (24) hours on paper facades, up to four (4) hours on copper materials and about three (3) to four (4) days on metals, stainless steel and it survives for very different time periods on latex and plastics.

By 26 September, there were 1,450,048 cases in Africa with 35,299 deaths[5]. In fact, the Africa region was touched relatively late by COVID-19 and has recorded far fewer cases compared to Asian, Europeans and the Americans (North and South)[6]. In the work of Rym Lalaoui et al. [7] they explain this slow propagation of the virus in Africa by the relatively young and rural population, the genetic polymorphism of the ACE2 receptor, cross-immunity and the use of antimalarial drugs. However, we should keep in mind that the COVID-19 diagnostic capacity remains limited in the Africa region. Thus, the reported numbers of COVID-19 cases and deaths are likely an underestimation of the true disease burden.

We aimed to evaluate the impact of COVID-19 on the clinical outcome of patients with cirrhosis. Since data on COVID-19 in patients with cirrhosis are lacking, we reported in this article a case of a patient affected with cirrhosis and SARS-CoV-2 infection in our hospital and we performed a multicentre retrospective study to describe the demographic, clinical and biochemical characteristics of patients with cirrhosis and SARS-CoV-2 infection in the literature. Through this work, we aim to evaluate the impact of COVID-19 on the clinical outcome of patients with cirrhosis.

\section{Observation}

65-year-old patient, hospitalized in our training for ascites of great abundance revealed by an abdominal distension evolving for 1 month without stopping of stool and gas and without other associated digestive signs in particular no digestive hemorrhage, no abdominal pain and no jaundice the whole evolving in a context of febrile sensation and asthenia. At the clinical examination we found a conscious patient, stable on the $\mathrm{HD}$ and respiratory level, $\mathrm{BMI}=23.9 \mathrm{Kg} / \mathrm{m}^{2}$, Conjunctiva slightly discolored, no jaundice, edema of both legs white painless , BU: Protein 1x , collateral 
venous circulation épigastric, and grade 3 ascites, flow sign + ; Liver and spleen difficult to appreciate; MA + TR examination: without abnormalities.

An exploratory puncture of the ascites fluid was made urgently in the context of eliminating a infection of ascites fluid which objective Aspect: young citrus, with a rate of Proteins $=6 \mathrm{~g} / 1$ Cyto Bacteriology $=\mathrm{GB}=120(\mathrm{PNN}=84$ lympho = 38.4); Red blood cell $=32$ and a sterile culture, biologically demonstrated, microcytic hypochromic anemia NFS: HB: 9g / dl; GB 5450; PQ 196000; ferritin: 26; CRP 22.8 hepato-cellular insufficiency: Albumin: 20.9, TP 42.2\%; without cytolysis or cholestasis GGT: 24 ALAT: 26 AST: 41 PAL 86 BT: 12.7 ; no ionic cloudiness $\mathrm{Na}+: 135 \mathrm{~K}+$ : 4.5; correct renal function: Urea 0.38 Creatinine: 8; Normal tumor markers (AFP: AFP $=4.82$ ) and $24 \mathrm{~h}$ proteinuria: $0.05 \mathrm{mg} / \mathrm{l}$.

A negative HVB serology with a positive HVC serology at a viral load at 1,650,262 IU / ml, a PCR of COVID 19 was done in front of the worsening of asthenia with febrile peaks which was positive and a thoracic CT showed pulmonary viral impairment estimated at $10 \%$. In parallel, the patient benefited from an abdominal ultrasound which objective a chronic hépathopatic liver with nodule in favor of hepatocellular carcinoma ; a complement by an angio MRI was made in favor of dysmorphic liver of chronic hepatopathy sits at the level of the segment II of an oval mass in isosignal T1 isosignal T2 hyper signal diffusion with intermediate ADC, encapsulated measuring $38 \times 35 \mathrm{~mm}$ in diameter, centered by a central zone in $\mathrm{T} 2$ hypersignal which takes the contrast, The rest of the mass took the contrast quickly in the arterial phase with homogenization at the early portal phase and Wash or at the time late portal. In all, this is a patient who presented an ascites decompensation of his cirrhosis child C10 and Meld 14 after viral hepatitis C with a nodule in favor of hepatocellular carcinoma.

Regarding the management of ascites: an evaluative puncture was performed with albumin infusion, and then the patient began diuretics after eliminating the contraindications, with good efficacy and tolerance, and without forgetting the antibiotic prophylaxis as part of the prevention of secondary infection of ascites fluid.

The hepato-cellular carcinoma was classified AFP Score 1, OKUDA: Stage: II, BCLC D advanced stage; the management of his carcinoma was resumed after treatment of covid 19 and drying of ascites. Thus, faced with the absence of any notion of digestive hemorrhage reported by the patient, a digestive fibroscope to search for esophageal and gastric varices was reported given the high risk of contamination.

According to the national protocol for the management of patients with COVID 19, treatment was instituted with chloroquine, azithromycin and paracetamol, after eliminating the contraindications, and isolation of the patient. Regular monitoring did not show any clinical or paraclinical complications. Therefore, the patient was discharged and told to stay confined at home for 14-day with respect for sanitary measures.

\section{DISCUSSION}

The mortality of COVID 19 have been rated between 2-6\%, and become more higher with underlying comoridities. Hepatic dysfunction has been seen in $14-53 \%$ of the patients, particularly in those with severe disease [8]. Moreover, the study of Massimo Iavarone et al. [9] showed that the infection with covid 19 led to rapid clinical deterioration. In fact, they noticed that 25 patients with Child-Pugh and low MELD score experienced a rapid deterioration in their liver function once they get the COVID 19 virus [9].

Pre-existing liver disease does not appear to be current in patients with coronavirus disease. Actually, they make up less than $1 \%$ of the reported case $[10,11]$. On the other hand, the presence of hepatic defiance increases the rate of mortality in COVID 19 patients. This was shown in the study of Xiaolong et al. [12], where in 21 consecutive patients with pre-existing cirrhosis, 5 did not survive covid 19 -infection and, specifically, patients with Child-Pugh class C. Another case series from Italy done by Massimo et al. [9] documented 50 patients with cirrhosis and COVID-19. They showed that $26 \%$ of these patients presented a model of end-stage liver disease (MELD score > 15). Nevertheless, the end-stage-liver disease was considered as the cause of death in only 5 patients, whilst respiratory failure due to COVID-19 accounted for death in 12 patients $[12,13]$. These studies outline that the Managing of a serious chronic liver disease in the midst of a global infectious pandemic is difficult and that we should change our approach therapy (figure $1)$. 


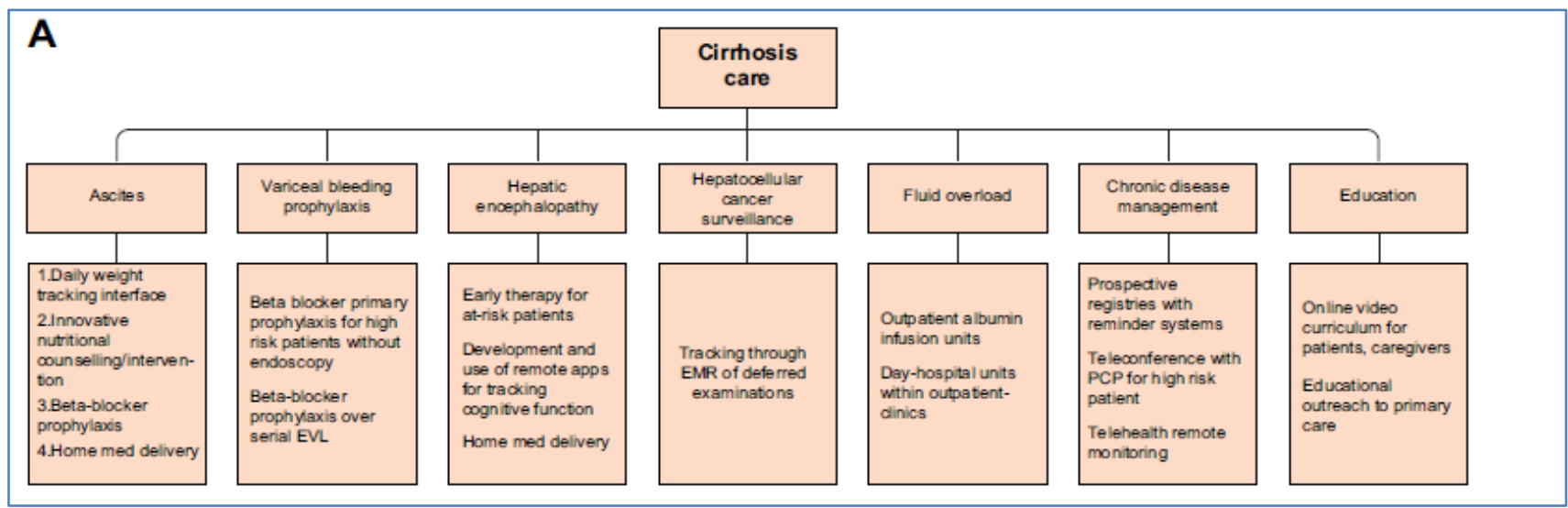

Fig-1: Cirrhosis care for patient with infection COVID 19 [14]

In the lights of these finding, we propose the following therapy: the means of prevention must be privileged, in order to reduce the need for therapeutic gestures. The hepatology care team should focus on proactive coordination, and offering secondary services as nutrition and vaccination which could reduce the burden of cirrhotic complications[14]. Moreover, the simplest conceptually change is the use of non-selective beta-blockers. However, in the shortest time the use of endoscopy is more recommended. Furthermore, nonselective beta-blockers reduce the risk of cirrhotic complications, unless large varices or bleeding stigmata were discovered in an emergent endoscopy[15]. As in cirrhotic patients with COVID-19, endoscopy was performed only in life-threatening. Once the patients are proposed for endoscopic procedures, they should be previously tested for COVID 19, through an RT-PCR nasopharyngeal swab test. Then, the health professionals should always use protective equipment during procedures[16]. Additionally, we recommend the use of blood markers (fibrotest) instead of the fibroscan. This procedure will reduce the contact with the COVID 19 patients. Moreover, the intravenous albumin infusions may help prevent readmissions and improve survival in COVID 19 patients with ascites and anasarca complications[17]. As to encephalopathy complications, they can be reduced by early recognition and therapeutic prevention by the care team. Their attention should be focused in education (e.g. nutrition), caregiver support, and early interventions to ensure medication compliance and to prevent decompensation for immunosuppressed and post-discharge patients. Thereby, telehealth through video visits has also been shown to improve the waiting time of potential transplant candidates and reduce referrals and travel for non-candidates. Actually, transplant evaluation can proceed without face-to-face visits [18, 19].

As to patients newly diagnosed with chronic HBV infection who develop COVID 19 superinfection, it would be reasonable to initiate a Current first-line nucleoside analogues therapy. In fact, the international guidelines reported that the Current first-line nucleoside analogues show an excellent long-term safety. In general, this treatment presents no drug interaction with the therapeutic options of COVID 19. Then, there is no need for stopping the antiviral treatment[20].

Furthermore, the use of chloroquine, hydroxychloroquine, and ribavirin is generally safe as no obvious drug-drug interactions have been reported [20]. As most COVID-19 therapies do not have considerable drug-drug interactions with direct-acting antiviral. Therefore, these treatments should be continued to maximise the sustained virological response rate. Premature cessation of direct-acting antiviral therapy might reduce this rate from nearly $100 \%$ to $74 \%[21]$. Any use of COVID-19 therapies in patients who are receiving direct-acting antiviral therapy should be closely monitored for potential adverse events. Because hepatitis flare related to viral hepatitis $\mathrm{c}$ following direct-acting antiviral therapy termination is very rare. This treatment might be stopped prematurely if there are problematic drug-drug interactions, or if patients are in critical condition (eg, under positive pressure ventilation or intubated)[22].

Management of hepatocellular carcinoma during the COVID-19 pandemic have been discussed by various oncology societies[23]. Because most patients with COVID-19 recover within 3-4 weeks[24], it would be reasonable to defer treatment of hepatocellular carcinoma for a few weeks after the recovery of COVID-19. Among the various treatment methods for hepatocellular carcinoma, surgical resection would be affected the most during the COVID-19 pandemic. On one hand, the demand to expand ventilation capacity for patients with COVID-19 has resulted in a shortage of anaesthetists to support surgery and other procedures such as ablation, which require a general anaesthetic. On the other hand, spreading COVID-19 aerosol by general anaesthesia in patients with confirmed or undiagnosed COVID-19 might pose a risk to health-care personnel in the operating theatre. Bridging therapy with transarterial chemoembolisation, radiofrequency ablation, or systemic chemotherapy might be offered in place of potentially curative interventions. Coupling this therapy 
with close monitoring, such as imaging and $\alpha$ fetoprotein, should be used to reduce the risk of patients progressing beyond criteria for transplantation, resection, or ablation [20].

\section{Conclusion}

Managing the pandemic of a serious chronic disease in the midst of a global infectious pandemic is difficult. Change will be needed, much more data are needed, and it is incumbent upon the entire healthcare establishment. The potential drug-drug interactions and liver toxicity must be thoroughly evaluated. Recommendations might need to be updated when a vaccine for COVID-19 becomes available, and safety and efficacy of such a vaccine has to be proven in patients with advanced liver disease and in recipients of liver transplant.

\section{REFERENCES}

1. Coccia M. Factors determining the diffusion of COVID-19 and suggested strategy to prevent future accelerated viral infectivity similar to COVID. Science of the Total Environment. 2020 Aug 10;729:138474.

2. Coccia M. Two mechanisms for accelerated diffusion of COVID-19 outbreaks in regions with high intensity of population and polluting industrialization: the air pollution-to-human and human-to-human transmission dynamics. MedRxiv. 2020 Jan 1.

3. Pyankov OV, Bodnev SA, Pyankova OG, Agranovski IE. Survival of aerosolized coronavirus in the ambient air. Journal of aerosol science. 2018 Jan 1; 115:158-63.

4. Leung WW, Sun Q. Charged PVDF multilayer nanofiber filter in filtering simulated airborne novel coronavirus (COVID-19) using ambient nanoaerosols. Separation and purification technology. 2020 Aug 15;245:116887.

5. CDC A. Coronavirus Disease 2019 (COVID-19). 2020.

6. Adekunle IA, Tella SA, Oyesiku KO, Oseni IO. Spatio-temporal analysis of meteorological factors in abating the spread of COVID-19 in Africa. Heliyon. 2020 Aug 1;6(8):e04749.

7. Lalaoui R, Bakour S, Raoult D, Verger P, Sokhna C, Devaux C, Pradines B, Rolain JM. What could explain the late emergence of COVID-19 in Africa?. New Microbes and New Infections. 2020 Sep 22:100760.

8. Jothimani D. COVID-19 and Liver. Journal of hepatology, 2020.

9. Iavarone M, D'Ambrosio R, Soria A, Triolo M, Pugliese N, Del Poggio P, Perricone G, Massironi $\mathrm{S}$, Spinetti A, Buscarini E, Viganò $\mathrm{M}$. High rates of 30-day mortality in patients with cirrhosis and COVID-19. Journal of hepatology. 2020 Nov $1 ; 73(5): 1063-71$.
10. CDC COVID-19 Response Team, CDC COVID-19 Response Team, CDC COVID-19 Response Team, Chow N, Fleming-Dutra K, Gierke R, Hall A, Hughes M, Pilishvili T, Ritchey M, Roguski K. Preliminary estimates of the prevalence of selected underlying health conditions among patients with coronavirus disease 2019-United States, February 12-March 28, 2020. Morbidity and Mortality Weekly Report. 2020 Apr 3;69(13):382-6.

11. Williamson EJ, Walker AJ, Bhaskaran K, Bacon S, Bates C, Morton CE, Curtis HJ, Mehrkar A, Evans D, Inglesby P, Cockburn J. OpenSAFELY: factors associated with COVID-19 death in 17 million patients. Nature. 2020 Jul 8.

12. Qi X, Liu Y, Wang J, Fallowfield JA, Wang J, Li X, Shi J, Pan H, Zou S, Zhang H, Chen Z. Clinical course and risk factors for mortality of COVID-19 patients with pre-existing cirrhosis: a multicentre cohort study. Gut. 2021 Feb 1;70(2):433-6.

13. Boettler T, Marjot T, Newsome PN, Mondelli MU, Maticic M, Cordero E, Jalan R, Moreau R, Cornberg M, Berg T. Impact of COVID-19 on the care of patients with liver disease: EASL-ESCMID position paper after 6 months of the pandemic. JHEP Reports. 2020 Oct;2(5).

14. Tapper EB, Asrani SK. COVID-19 pandemic will have a long-lasting impact on the quality of cirrhosis care. Journal of hepatology. 2020 Apr 13.

15. Villanueva $\mathrm{C}$, Albillos $\mathrm{A}$, Genescà $\mathrm{J}$, Garcia-Pagan JC, Calleja JL, Aracil C, Bañares R, Morillas RM, Poca M, Peñas B, Augustin S. $\beta$ blockers to prevent decompensation of cirrhosis in patients with clinically significant portal hypertension (PREDESCI): a randomised, double-blind, placebo-controlled, multicentre trial. The lancet. 2019 Apr 20;393(10181):1597-608.

16. Garrido I, Liberal R, Gaspar R, Macedo G. Cirrhosis management in a major referral center during COVID-19. JHEP Reports. 2020 Oct 1.

17. Caraceni P, Riggio O, Angeli P, Alessandria C, Neri S, Foschi FG, Levantesi F, Airoldi A, Boccia S, Svegliati-Baroni G, Fagiuoli S. Long-term albumin administration in decompensated cirrhosis (ANSWER): an open-label randomised trial. The Lancet. 2018 Jun 16;391(10138):2417-29.

18. John BV, Love E, Dahman B, Kurbanova N, Konjeti VR, Sundaram LT, Deng Y, Aubuchon S, Heuman D, Bajaj JS, Gilles H. Use of telehealth expedites evaluation and listing of patients referred for liver transplantation. Clinical gastroenterology and hepatology. $2020 \mathrm{Jul}$ 1;18(8):1822-30.

19. Konjeti VR, Heuman D, Bajaj JS, Gilles H, Fuchs M, Tarkington P, John BV. Telehealth-based evaluation identifies patients who are not candidates for liver transplantation. Clinical Gastroenterology and Hepatology. 2019 Jan $1 ; 17(1): 207-9$.

20. Lai-Hung Wong G, Wai-Sun Wong V, Thompson A, Jia J, Hou J, Lesmana A, Rinaldi C, Susilo A, Tanaka Y, Chan WK, Gane E. Management of 
patients with liver derangement during the COVID19 pandemic: an Asia-Pacific position statement.

21. Marshall MC, Herrera JL. Lack of patient compliance in real-world practice negatively affects sustained viral response rates to direct acting agent therapy for hepatitis C. Digestive diseases and sciences. 2018 Dec;63(12):3228-32.

22. Huang $\mathrm{CF}, \mathrm{Yu} \mathrm{ML}$. Unmet needs of chronic hepatitis $\mathrm{C}$ in the era of direct-acting antiviral therapy. Clinical and molecular hepatology. 2020 Jul;26(3):251.

23. Online TILCAI. Management of hcc during covid19: ilca guidance. 2020.

24. Huang C, Wang Y, Li X, Ren L, Zhao J, Hu Y, Zhang L, Fan G, Xu J, Gu X, Cheng Z. Clinical features of patients infected with 2019 novel coronavirus in Wuhan, China. The lancet. $2020 \mathrm{Feb}$ 15;395(10223):497-506. 